\title{
Oral Health Status and Oral Impact on Daily Performance in Elderly in Northeastern Region Thailand
}

\author{
By K. Sombateyotha ${ }^{1}$, U. Mahaweerawat ${ }^{1}$
}

\begin{abstract}
The quality of life index as oral impact on daily performance (OIDP) is very significant indicator for people teeth loss or oral health problem in specific socio-cultural community where generalized for assessment. The research is aimed to investigate dental health status and their OIDP for the elderly. This cross-sectional descriptive study was comprised of seven provinces by randomized selected in Northeastern region of Thailand, with 2,939 elderly. All those elderly were dental health examined and interviewed by using standardized questionnaire included dental health behavior, psychological health and OIDP form. This results showed that the OIDP scoring 5 level, first 3 of 5 level ranking is the most severity level magnitude such as eating food $(52.2 \%)$, speaking $(51.9 \%)$, embarrassed by teeth appearance $(51.9 \%$ ), which affected daily life performances. The statistical analysis showed that subjects perceived their irritation in oral cavity [adjusted odds ratio $(\mathrm{AOR})=2.11 ; 95 \% \mathrm{CI}=1.62-4.31$ ], xerostomia $[\mathrm{AOR}=1.89 ; 95 \% \mathrm{CI}=1.02-3.13]$, restricted their sugar consumption $[\mathrm{AOR}=2.38$; $95 \% \mathrm{CI}=1.22-3.80]$, low frequency of cleaning teeth $[\mathrm{AOR}=1.96 ; 95 \% \mathrm{CI}=1.09-3.03]$, visited a dentist during the past three years or more $[\mathrm{AOR}=2.89 ; 95 \% \mathrm{CI}=1.20-4.15]$. In conclusions, the high prevalence of oral hygienic problem with OIDP also still high magnitude level. Thus community oral health education programs and dental skill training need to be improved and fulfilled.
\end{abstract}

Keyword: Oral impact on daily performance (OIDP), elderly, oral health

\section{Introduction}

Thailand is going up to "aging society". Healthy elderly is mean whose had both physical health condition and mental health condition including healthy oral condition Peterson (2003) suggested 3 aspect for better oral health promotion such as 1) oral health is the most composition of general health 2) oral health was related in general health and 3) oral health is the most important indicator of quality of life. Several dimension of quality of life(QOL) were relation to oral health and general health. The completeness of teeth and oral organ is the important functional of chew, swallow, speaking, smiling, laughing and including important confident for human mental and social contact. (Srisilapanan, 1998). Socio-dental indicators was set up for examined of oral health related QOL, its including social aspect, perceived oral health condition. The OIDP index is the self-assessment level of its oral impacts (Adulyanon and Sheiham,1997), the OIDP was use to study and development tool for explored specific oral health problem thus it could divided into level of oral problem, therefore dental need is significant of service plan of dentist. (Gherunpong , 2007). All of reason this study was conducted oral health and its impacts on elderly daily living in namely OIDP. 
This research study is aimed at investigate for dental health status and their OIDP in the elderly.

\section{Material and Methods}

This cross-sectional analytical descriptive study was conducted during January to April 2016. The sample size were 2,939 elderly with 1,253 males and 1,687 females, who residing in 7 provinces such as Mahasarakham, Khonkaen, Nakhonratchasima, Buriram, Chaiyabpumi, Roi-et and Surin of northeastern region of Thailand, All subjects were willing to participate in interviewed and examined orally after giving written informed consent. The questionnaire was comprised of four parts. Part I is personal information such as sex, age, education, marital status, income, visited a dentist history. Part II is comprised of general health status and psychological health status information such as ever present illness, perceived health status, drug use and perceived physical limitation and disability. Part III is oral health behaviors such as cleaning teeth, cleaning denture, risk behaviors; cigarette smoking, chew betel palm, visited a dentist during the past, sugary food consumption. Part IV comprised of oral health status and its impact on daily performance by using the OIDP form developed by Adulyanon and Sheiham(1997), this tool included 8 aspects such as eating and enjoying food, speaking, cleaning teeth, sleeping, embarrassed by teeth appearance, maintaining emotional stability, working and contact with people. The psychosocial health status were evaluated with 7 aspects, namely sadly, bored, crying, worried, moody, apathy, hopeless, and each question on the psychosocial aspect all impacted. All subjects were asked how frequently they had experienced the problem during last month. All responses were recorded using a 3-point Likert Scale as point 3= never occur for all 7 days a week, 2=sometime ever within 4 days a week, $1=$ happened for all 7 days a week. Total score were calculated of 7 items, score 17 and over mean free for depression status, score less than 17 mean present of depression status. The impact on oral conditions and QOL was assessed through the OIDP followed from by Adulyanon and Sheiham(1997), the data gathering for past six months as oral health, dental and denture condition, while get high score interpreted as magnitude of problem. Each performance score of OIDP calculated by multiplied with frequency (0-5point) and severity score (0-5point), then were summed up for 10 performances score. Finally the overall OIDP score was derived from sum all score and divided by maximum possible score ( 5 frequency x 5 severity scores x10 performances) and multiplied with 100 for per-centage score. In addition to the OIDP score, the extension of OIDP impacts was also reported, this is an alternative mean to quantifying oral impacts, previously suggested for the OIDP (Robinson et al., 2003) and refers to the number of OIDP performances with impacts affecting a respondent's quality of life over the past six months. The OIDP performances score is divided into 2 categories by using $50^{\text {th }}$ per-centile which OIDP score $=0$ refer to free of oral condition impact on OIDP, if score $>0$ refer to present of impact on OIDP oral condition.

\section{Results}

All of 2,939 elderly, 1,252 males (42.6\%), 1,687 females (57.4\%), age mean 65.6 
years $(\mathrm{SD}=4.33)$ ranged from 60-86 years old. The Sixty-five percent of subject had educational level as primary school, 63.7\% were agriculturalist, 57.5\% family income average 1,001-5,000 baht monthly ( 1 baht=33 US\$), 72.4\% visited a dentist within the previous year, $63.4 \%$ no illness, $36.6 \%$ illness conditions of high blood pressure $(15.8 \%)$, diabetic $(10.7 \%)$, joint wrist or knee difficulty movement $(2.7 \%)$, depressive $(16.3 \%)$, 55.8 per-cent with 0-12 teeth lose, 13-20 teeth lose (21.5\%), 21 and more teeth lose $(22.7 \%), 72.7 \%$ had experienced dental caries, no denture status $(72.7 \%)$, partial removable denture of $4.1 \%$, total removable denture of $12.2 \%$. The subjects were cleaning teeth--once a day (59.4\%), twice a day (29.1\%) respectively, restricted sugar consumption $33.7 \%$, no smoking $67.4 \%$, smoking $32.6 \%$, chew betel palm $25.3 \%$, had visited a dentist previous year $(73.8 \%)$.

The magnitude OIDP in the elderly found that eating $(52.2 \%)$, speaking $(51.9 \%)$, embarrassed by teeth appearance $(51.9 \%)$, cleaning teeth or denture $(31.2 \%)$ with affected performances, frequency of oral impact on embarrassed by teeth appearance (mean=3.63), speaking (mean 3.21), maintaining emotional stability $($ mean $=3.41)$ (Table 1). The causes of oral problem such as teeth lose $(14.7 \%)$, teeth pain $(10.1 \%)$, teeth mobility $(7.8 \%)$, bad breath $(6.9 \%)$. As shown in Table1

Table 1. Frequency and Severity oral impact of its OIDP $(n=2,939)$

\begin{tabular}{lccc}
\hline Impact & $\begin{array}{c}\text { Number } \\
\text { (per-centage) }\end{array}$ & $\begin{array}{c}\text { Frequency of impact } \\
\text { level (0-5) } \\
\text { Mean (SD) }\end{array}$ & $\begin{array}{c}\text { Severity of impact } \\
\text { level (0-5) } \\
\text { Mean (SD) }\end{array}$ \\
\hline Eating & $1,534(52.2)$ & $2.05(1.14)$ & $2.85(1.27)$ \\
Speaking & $1,525(51.9)$ & $3.21(2.22)$ & $1.68(2.11)$ \\
Embarrassed by the teeth appearance & $1,525(51.9)$ & $3.63(1.26)$ & $3.85(1.33)$ \\
Cleaning teeth or denture & $917(31.2)$ & $2.15(1.55)$ & $2.67(1.23)$ \\
Contact with people & $664(22.6)$ & $2.54(1.18)$ & $2.23(1.21)$ \\
Working & $517(17.6)$ & $1.82(1.20)$ & $2.28(1.66)$ \\
Maintaining emotional stability & $470(16.0)$ & $3.01(2.00)$ & $3.14(2.45)$ \\
Sleeping well & $350(11.9)$ & $1.23(1.83)$ & $3.41(2.42)$ \\
\hline
\end{tabular}

\section{Discussion}

The OIDP is one of the most widely used socio-dental indicators which focus on measuring serious oral impacts on a person's ability to perform daily activities (Gherunpong, 2007). The previous study by (Mcgrath et al., 2000) suggested that OIDP indicators might be performed in older person like many other QOL indicators in dentistry have focused primarily on older age groups, partly for the assumption that they would be a lifetime's experience of oral ill health and thus are likely to perceive oral health as having a greater impact on their QOL. This study showed the magnitude of oral impacts on eating $(52.2 \%)$ speaking $(51.9 \%)$ similar to previous study of Srisilapanan and Sheiham, 2001 (52.8\%), Charoentanyarak et al., (2014) (51.4\%), Wong et al., 2002 $(39.7 \%)$. In term of frequency of impacts on its OIDP were embarrassed by the teeth appearance (mean=3.63) followed by speaking (mean=3.21) similar to those found in Great Britain Sheiham et al., (2001). The most seriously oral impacts were embarrassed by the teeth appearance (mean=3.85) higher than previous study Charoentanyarak et al., 
(2014).

The relationship between oral impacts on daily performance and clinical status by using bivariate analysis founded significant association $(\mathrm{p} \leq 0.05)$ which was observed in oral impacts on daily performances with perceived their irritation in oral cavity, xerostomia, teeth lose, frequency of cleaning teeth, restricted their sugar consumption, cigarette smoking, and visited a dentist during the past three years or more, respectively. Multivariate logistic regression applied for considering association between dependent variables after controlling for age. The finding of five significant factors for probability with one or more impact were identified as perceived their irritation in oral cavity (adjusted OR 2.11, 95\% CI: 1.62-4.31), xerostomia (AOR 1.89, 95\%CI: 1.02-3.13), frequency of cleaning teeth (AOR 1.96, 95\% CI: 1.09-3.03), restricted their sugar consumption (AOR 2.38, 95\% CI: 1.22-3.80), visited a dentist during the past three years or more (AOR 2.89, 95\%CI: 1.20-4.15). (shown in table 2)

Table 2. Bivariate analysis and multivariable logistic regression model evaluating the relationship between the OIDP and selected variables

\begin{tabular}{lccc}
\hline \multirow{2}{*}{ Factors } & Bivariate analysis & \multicolumn{2}{c}{$\begin{array}{c}\text { Multivariable logistic } \\
\text { regression }\end{array}$} \\
\cline { 2 - 4 } & $\mathrm{OR}_{\text {crude }}(95 \% \mathrm{CI})$ & $\mathrm{OR}_{\text {adj }}(95 \% \mathrm{CI})$ & $p$-value \\
\hline Present of high blood pressure & $0.76(0.33-1.30)$ & - & \\
Perceived their irritation in oral cavity & $1.96(1.47-4.10)$ & $2.11(1.62-4.31)$ & 0.002 \\
Xerostomia & $1.65(1.02-3.00)$ & $1.89(1.02-3.13)$ & 0.016 \\
Dental caries & $1.10(0.82-2.10)$ & - & \\
Teeth lose & $1.59(1.04-2.72)$ & - & \\
Prosthetic need & $1.48(0.87-2.22)$ & - & \\
Frequency of cleaning teeth & $1.72(1.07-3.00)$ & $1.96(1.09-3.03)$ & 0.014 \\
Restricted their sugar consumption & $2.01(1.18-3.72)$ & $2.38(1.22-3.80)$ & 0.002 \\
Cigarette smoking & $1.15(1.04-2.61)$ & - & \\
visited a dentist during the past three years or more & $2.76(1.16-4.02)$ & $2.89(1.20-4.15)$ & 0.001 \\
\hline
\end{tabular}

The multivariable logistic regression found that the factors association with OIDP were perceived their irritation in oral cavity, xerostomia, frequency of cleaning teeth, restricted their sugar consumption, and visited a dentist during the past three years or more were similar to the study of Sanberg and Wikblad (2003) found as the diabetic patient who dissatisfaction with their teeth and mouth, feeling of dry mouth and poor financial status afforded for one-fourth of the explanation above of the variance in all health domains. The possible limitation of this study is cross-sectional nature which poses problems in relation to hypothesis testing since data on risk factors and outcome are assessed at the same time, but this particular issue does not seem to affect our result. However, future longitudinal studies are need to better understand.

In conclusions, the high prevalence of oral hygienic problem with OIDP also still high magnitude level. Thus community oral health education programs and dental health care skill training need to be improved and fulfilled by community dental health care personnel or public health dentist. 


\section{References}

Adulyanon S., Sheiham A., (1997) Oral impacts on daily performances. In G.D. Slade (Ed.), Measuring oral health and quality of life. Chapel Hill: University of North Carolina, pp. 151-60.

Charoentanyarak S., Khiewyoo J., Weraarchakul W., (2014) Oral bealth related quality of life among diabetes mellitus patients: A case study in Suwannakuba District, Nongbualumphu Province. Srinagarind Med J, 29(4), pp. $339-344$.

Gherunpong S., (2007) Oral health-related quality of life: Part II Index for adults. Review article. Journal of Health Science, 16(2), March-April, pp. 306-317.

Mcgrath C., Bedi R., Gilthorpe MS. (2000) Oral health related quality of life--views of the public in the United Kingdom. Community Dent Health, 17, pp. 3-7.

Ngoenwiwatkul Y., Chatrchaiwiwatana S., and Chaiprakarn K., (2014) Dental status and its impact on the quality of life of elderly in Phon Sawan, Nakhon Phanom Province. Southeast Asian J Trop Med Public Health, 45(1), January, pp. 236-243.

Peterson PE., (2013) The World Oral Health report 2003: continuous improvement of Oral Health in the 21st century-the approach of the WHO Global Oral Health Pramme. Community Dent oral epidemiol, 32 Suppl 1: 3-24.

Sandberg GE., Wikblad KF. (2003) Oral health and health-related quality of life in type 2 diabetic patients and nondiabetic controls. Acta Odontal Scand, 61(3), pp. 141-148.

Sheiham A., Steele JG., Marcenes W., Tsakos G., Finch S., and Walls AMG., (2001) Prevalence of impacts of dental and oral disorders and their effects on eating among older people; a national survey in Great Britain. Community Dental Oral Epidemiol, 29, pp. 125-203.

Srisilapanan P., Sheiham A., (2001) The prevalence of dental impacts on daily performances in older people in Northern Thailand. Gerontology, 28, pp. 102-108.

Suliman NM., Johannessen AC., Ali RW., Salman H., Astrom AN. (2012) Influence of oral mucosal lesions and oral symptoms on oral health related quality of life in dermatological patients: a cross-sectional study in Sudan. BMC Oral Health, 12, pp. 19-30.

Wong MC., Lo EC., McMillan AS. (2002) Validation of a Chinese version of the Oral Health Impact Profile (OHIP). Community Dent Oral Epidemiol, 30, pp. 423-430. 